\title{
A Presentation of the Kähler Differential Module for a Fat Point Scheme in $\mathbb{P}^{n_{1}} \times \cdots \times \mathbb{P}^{n_{k}}$
}

\author{
Tran N. K. Linh ${ }^{1,3, *}$, Elena Guardo ${ }^{2, * *}$, and Le Ngoc Long ${ }^{3, * * *}$ \\ ${ }^{1}$ Department of Mathematics, Hue University of Education, 34 Le Loi, Hue, Vietnam \\ ${ }^{2}$ Dipartimento di Matematica e Informatica, Viale A. Doria 6, 95100 - Catania, Italy \\ ${ }^{3}$ Fakultät für Informatik und Mathematik, Universität Passau, D-94030 Passau, Germany
}

\begin{abstract}
Let $\mathbb{Y}$ be a fat point scheme in $\mathbb{P}^{n_{1}} \times \cdots \times \mathbb{P}^{n_{k}}$ over a field $K$ of characteristic zero. In this paper we introduce the multi-graded Kähler differential module for $\mathbb{Y}$ and we establish a short exact sequence of this module in terms of the thickening of $\mathbb{Y}$.
\end{abstract}

\section{Introduction}

In [1], G. de Dominicis and M. Kreuzer introduced some methods using algebraic differential forms into the study of finite sets of points in the projective $n$-space $\mathbb{P}^{n}$ over a field $K$ of characteristic zero. Explicitly, given a finite set of points $\mathbb{X}$ in $\mathbb{P}^{n}$ with homogeneous vanishing ideal $I_{\mathbb{X}}$ in $R=K\left[X_{0}, \ldots, X_{n}\right]$ and homogeneous coordinate ring $R_{\mathbb{X}}=R / I_{\mathbb{X}}$, the Kähler differential module for $\mathbb{X}$ is the $R_{\mathbb{X}}$-module $\Omega_{R_{\mathbb{X}} / K}^{1}=J / J^{2}$ where $J$ is the kernel of the multiplication map $\mu: R_{\mathbb{X}} \otimes_{K} R_{\mathbb{X}} \rightarrow R_{\mathbb{X}}$. One of interesting results in [1] is the canonical exact sequence for the Kähler differential module

$$
0 \rightarrow I_{\mathbb{X}}^{(2)} / I_{\mathbb{X}}^{2} \rightarrow I_{\mathbb{X}} / I_{\mathbb{X}}^{2} \rightarrow R_{\mathbb{X}}^{n+1}(-1) \rightarrow \Omega_{R_{\mathbb{X}} / K}^{1} \rightarrow 0
$$

Based on this exact sequence, the structure of this module can be precisely described in several special cases, for instance, if $\mathbb{X}$ is the complete intersection of hypersurfaces of degrees $d_{1}, \ldots, d_{n}$ then it follows that the Hilbert function of $\Omega_{R_{\mathrm{X}} / K}^{1}$ is given by $\mathrm{HF}_{\Omega_{R_{\mathbb{X} / K}}^{1}}(i)=$ $(n+1) \mathrm{HF}_{\mathbb{X}}(i-1)-\sum_{j=1}^{n} \operatorname{HF}_{\mathbb{X}}\left(i-d_{j}\right)$ for all $i \in \mathbb{Z}$. Later, in [2, 4], the differential algebra techniques were extended to fat point schemes in $\mathbb{P}^{n}$ and in $\mathbb{P}^{1} \times \mathbb{P}^{1}$.

In this paper we will consider the natural question of whether these differential algebraic methods can be applied to study fat point schemes of a multiprojective space $\mathbb{P}^{n_{1}} \times \cdots \times \mathbb{P}^{n_{k}}$ and, especially, we look closely at the generalization of the above canonical exact sequence to fat point schemes in $\mathbb{P}^{n_{1}} \times \cdots \times \mathbb{P}^{n_{k}}$. Let $\mathbb{Y}$ be a fat point scheme in $\mathbb{P}^{n_{1}} \times \cdots \times \mathbb{P}^{n_{k}}$ with multihomogeneous vanishing ideal $I_{\mathbb{Y}} \subseteq S=K\left[X_{10}, \ldots, X_{1 n_{1}}, \ldots, X_{k 0}, \ldots, X_{k n_{k}}\right]$ and let $R_{\mathbb{Y}}=S / I_{\mathbb{Y}}$ be the multi-graded coordinate ring of $\mathbb{Y}$. We show that the Kähler differential module $\Omega_{R_{Y} / K}^{1}$ admits the following exact sequence.

\footnotetext{
*e-mail: tnkhanhlinh141@ gmail.com

**e-mail: guardo@dmi.unict.it

***e-mail: nglong16633@gmail.com
} 
Theorem 1.1. (Theorem 3.5) Let $\mathbb{Y}=m_{1} P_{1}+\cdots+m_{s} P_{s}$ be a fat point scheme in $\mathbb{P}^{n_{1}} \times \cdots \times \mathbb{P}^{n_{k}}$, and let $\mathbb{V}=\left(m_{1}+1\right) P_{1}+\cdots+\left(m_{s}+1\right) P_{s}$ be the thickening of $\mathbb{Y}$. Then we have the exact sequence of $\mathbb{Z}^{k}$-graded $R_{\mathbb{Y}}$-modules

$$
0 \longrightarrow I_{\mathbb{Y}} / I_{\mathbb{V}} \longrightarrow R_{\mathbb{Y}}^{n_{1}+1}\left(-e_{1}\right) \oplus \cdots \oplus R_{\mathbb{Y}}^{n_{k}+1}\left(-e_{k}\right) \longrightarrow \Omega_{R_{\mathbb{Y}} / K}^{1} \longrightarrow 0 .
$$

This result shows that one can compute the Hilbert function of the Kähler differential module for $\mathbb{Y}$ from the Hilbert functions of $\mathbb{Y}$ and $\mathbb{V}$, in particularly, to compute the Hilbert function $\mathrm{HF}_{\Omega_{R_{\mathrm{Y}} / K}^{1}}(\underline{i})$, we need to compute $\mathrm{HF}_{\Omega_{R_{\mathrm{Y}} / K}^{1}}(\underline{i})$ for only a finite number of $\underline{i} \in \mathbb{Z}^{k}$.

\section{Basic Facts and Notation}

Let $K$ be a field of characteristic zero. Let $k \geq 2$ be a positive integer, let $i$ denote the tuple $\left(i_{1}, \ldots, i_{k}\right) \in \mathbb{Z}^{k}$, and let $|\underline{i}|=\sum_{l} i_{l}$. We write $\underline{i} \leq \underline{j}$ if $i_{l} \leq j_{l}$ for every $l=\overline{1}, \ldots, k$. Also, let $\left\{e_{1}, \ldots, e_{k}\right\}, e_{i}=(0, \ldots, 1, \ldots, 0)$, be the canonical basis of $\mathbb{Z}^{k}$.

The multi-graded coordinate ring of $\mathbb{P}^{n_{1}} \times \cdots \times \mathbb{P}^{n_{k}}$ is the the polynomial ring $S=$ $K\left[X_{10}, \ldots, X_{1 n_{1}}, \ldots, X_{k 0}, \ldots, X_{k n_{k}}\right]$ equipped with the $\mathbb{Z}^{k}$-grading defined by $\operatorname{deg} X_{i 0}=\cdots=$ $\operatorname{deg} X_{i n_{i}}=e_{i}$ for $i=1, \ldots, k$. For $\underline{i}=\left(i_{1}, \ldots, i_{k}\right) \in \mathbb{Z}^{k}$, we let $S_{\underline{i}}$ be the homogeneous component of degree $\underline{i}$ of $S$, i.e., the $K$-vector space with basis

$$
\left\{X_{10}^{\alpha_{10}} \cdots X_{1 n_{1}}^{\alpha_{1 n_{1}}} \cdots X_{k 0}^{\alpha_{k 0}} \cdots X_{k n_{k}}^{\alpha_{k n_{k}}} \mid \sum_{l=1}^{n_{j}} \alpha_{j l}=i_{j}, \alpha_{j l} \in \mathbb{Z}\right\} .
$$

Given an ideal $I \subseteq S$, we set $I_{i}:=I \cap S_{\underline{i}}$ for all $\underline{i} \in \mathbb{Z}^{k}$. Clearly, $I_{\underline{i}}$ is a $K$-vector subspace of $S_{\underline{i}}$ and $I \supseteq \bigoplus_{i \in \mathbb{Z}^{k}} I_{\underline{i}}$. The ideal $I$ is called $\mathbb{Z}^{k}$-homogeneous if $I=\bigoplus_{i \in \mathbb{Z}^{k}} I_{\underline{i}}$. If $I$ is a $\mathbb{Z}^{k}$ homogeneous ideal of $S$ then the quotient ring $S / I$ also inherits the structure of a multi-graded $\operatorname{ring}$ via $(S / I)_{\underline{i}}:=S_{\underline{i}} / I_{\underline{i}}$ for all $\underline{i} \in \mathbb{Z}^{k}$.

A finitely generated $S$-module $M$ is a $\mathbb{Z}^{k}$-graded $S$-module if it has a direct sum decomposition

$$
M=\bigoplus_{\underline{i} \in \mathbb{Z}^{k}} M_{\underline{i}}
$$

with the property that $S_{\underline{i}} M_{\underline{j}} \subseteq M_{\underline{i}+\underline{j}}$ for all $\underline{i}, \underline{j} \in \mathbb{Z}^{k}$.

Definition 2.1. Let $M$ be a finitely generated $\mathbb{Z}^{k}$-graded $S$-module. The Hilbert function of $M$ is the numerical function $\mathrm{HF}_{M}: \mathbb{Z}^{k} \rightarrow \mathbb{Z}$ defined by

$$
\mathrm{HF}_{M}(\underline{i}):=\operatorname{dim}_{K} M_{\underline{i}} \text { for all } \underline{i} \in \mathbb{Z}^{k} .
$$

In particular, for a $\mathbb{Z}^{k}$-homogeneous ideal $I$ of $S$, the Hilbert function of $S / I$ satisfies

$$
\mathrm{HF}_{S / I}(\underline{i}):=\operatorname{dim}_{k}(S / I)_{\underline{i}}=\operatorname{dim}_{k} S_{\underline{i}}-\operatorname{dim}_{k} I_{\underline{i}} \text { for all } \underline{i} \in \mathbb{Z}^{k} .
$$

If $M$ is a finitely generated $\mathbb{Z}^{k}$-graded $S$-module such that $\operatorname{HF}_{M}(\underline{i})=0$ for $\underline{i} \nsucceq(0, \ldots, 0)$, we write the Hilbert function of $M$ as an infinite matrix, where the initial row and column are indexed by 0 .

A point in the space $\mathbb{P}^{n_{1}} \times \cdots \times \mathbb{P}^{n_{k}}$ has the form

$$
P=\left[a_{10}: a_{11}: \cdots: a_{1 n_{1}}\right] \times \cdots \times\left[a_{k 0}: a_{k 1}: \cdots: a_{k n_{k}}\right] \in \mathbb{P}^{n_{1}} \times \cdots \times \mathbb{P}^{n_{k}}
$$

where $\left[a_{j 0}: a_{j 1}: \cdots: a_{j n_{j}}\right] \in \mathbb{P}^{n_{j}}$. Its vanishing ideal is the bihomogeneous prime ideal of the form

$$
I_{P}=\left\langle L_{11}, \ldots, L_{1 n_{1}}, \ldots, L_{k 1}, \ldots, L_{k n_{k}}\right\rangle
$$


where $L_{j l}=a_{j l} X_{j 0}-a_{j 0} X_{j l}$ and $\operatorname{deg}\left(L_{j l}\right)=e_{j}$ for $l=1, \ldots, n_{j}$.

When $\mathbb{X}=\left\{P_{1}, \ldots, P_{s}\right\}$ is a set of $s$ distinct points in $\mathbb{P}^{n_{1}} \times \cdots \times \mathbb{P}^{n_{k}}$, then the vanishing ideal of $\mathbb{X}$ is $I_{\mathbb{X}}=I_{P_{1}} \cap \cdots \cap I_{P_{s}}$ and its $\mathbb{Z}^{k}$-graded coordinate ring is $R_{\mathbb{X}}=S / I_{\mathbb{X}}$.

Definition 2.2. Let $\mathbb{X}=\left\{P_{1}, \ldots, P_{s}\right\}$ is a set of $s$ distinct points in $\mathbb{P}^{n_{1}} \times \cdots \times \mathbb{P}^{n_{k}}$, and let $m_{1}, \ldots, m_{s}$ are positive integers. The ideal $I_{\mathbb{Y}}=I_{P_{1}}^{m_{1}} \cap \cdots \cap I_{P_{s}}^{m_{s}}$ defines a 0-dimensional subscheme $\mathbb{Y}$ of $\mathbb{P}^{n_{1}} \times \cdots \times \mathbb{P}^{n_{k}}$.

(a) The scheme $\mathbb{Y}$ is called a fat point scheme of $\mathbb{P}^{n_{1}} \times \cdots \times \mathbb{P}^{n_{k}}$ and is denoted by $\mathbb{Y}=$ $m_{1} P_{1}+\cdots+m_{s} P_{s}$.

(b) The number $m_{j}$ is called the multiplicity of the point $P_{j}$.

(c) The fat point scheme $\mathbb{V}=\left(m_{1}+1\right) P_{1}+\cdots+\left(m_{s}+1\right) P_{s}$ in $\mathbb{P}^{n_{1}} \times \cdots \times \mathbb{P}^{n_{k}}$ is called the thickening of $\mathbb{Y}$.

The support of $\mathbb{Y}$ is $\operatorname{Supp}(\mathbb{Y})=\mathbb{X}$, the vanishing ideal of $\mathbb{Y}$ is $I_{\mathbb{Y}}$ and its $\mathbb{Z}^{k}$-graded coordinate ring is the residue class ring $R_{\mathbb{Y}}=S / I_{\mathbb{Y}}$.

If $\mathbb{Y}$ is a fat point scheme in $\mathbb{P}^{n}$, then there exists a linear form that is a non-zerodivisor for the homogeneous coordinate ring of $\mathbb{Y}$. Using this property, the following lemma for fat point schemes in $\mathbb{P}^{n_{1}} \times \cdots \times \mathbb{P}^{n_{k}}$ follows similarly from [3, Lemma 1.2].

Lemma 2.3. Let $\mathbb{Y}$ be a fat point scheme of $\mathbb{P}^{n_{1}} \times \cdots \times \mathbb{P}^{n_{k}}$. Then for $i \in\{1, \ldots, k\}$ there exists $L_{i} \in S_{e_{i}}$ such that the image of $L_{i}$ in the residue class ring $R_{\mathbb{Y}}=S / I_{\mathbb{Y}}$ is a non-zerodivisor of $R_{\mathbb{Y}}$.

Remark 2.4. After a suitable change of coordinates, we can assume that $L_{i}=X_{i 0}$ for $i=$ $1, \ldots, k$. This also implies that $X_{i 0}$ does not vanish at any point of $\operatorname{Supp}(\mathbb{Y})=\mathbb{X}$. In this case if $x_{i j}$ denotes the image of $X_{i j}$ in $R_{\mathbb{Y}}$, then $x_{10}, \ldots, x_{k 0}$ are non-zerodivisors of $R_{\mathbb{Y}}$.

As a consequence of the lemma and [8, Proposition 1.9] we get several basis properties of the Hilbert function of $\mathbb{Y}$.

Theorem 2.5. Let $\mathbb{Y}=m_{1} P_{1}+\cdots+m_{s} P_{s}$ be a fat point scheme of $\mathbb{P}^{n_{1}} \times \cdots \times \mathbb{P}^{n_{k}}$ with Hilbert function $\mathrm{HF}_{\Psi}$, and let $j \in\{1, \ldots, k\}$ and $N=n_{1}+\cdots+n_{k}$.

(a) For all $\underline{i} \in \mathbb{Z}^{k}$ we have $\mathrm{HF}_{\mathbb{Y}}(\underline{i}) \leq \mathrm{HF}_{\mathbb{Y}}\left(\underline{i}+e_{j}\right) \leq \sum_{k=1}^{s}\left(\begin{array}{c}N+m_{k}-1 \\ m_{k}-1\end{array}\right)$.

(b) If $\mathrm{HF}_{\mathbb{Y}}(\underline{i})=\mathrm{HF}_{\mathbb{Y}}\left(\underline{i}+e_{j}\right)$ then $\mathrm{HF}_{\mathbb{Y}}(\underline{i})=\operatorname{HF}_{\mathbb{Y}}\left(\underline{i}+2 e_{j}\right)$.

\section{A Presentation of the Kähler Differential Module}

In the following we let $\mathbb{Y}$ be a fat point scheme in $\mathbb{P}^{n_{1}} \times \cdots \times \mathbb{P}^{n_{k}}$ supported at $\mathbb{X}$. We also denote the image of $X_{i j}$ in $R_{\mathbb{Y}}$ by $x_{i j}$. According to Remark 2.4, we may always assume that $x_{10}, \ldots, x_{k 0}$ are non-zerodivisors of $R_{\mathbb{Y}}$.

In the multi-graded algebra

$$
R_{\mathbb{Y}} \otimes_{K} R_{\mathbb{Y}}=\bigoplus_{\underline{i} \in \mathbb{Z}^{k}}\left(\bigoplus_{\underline{j}+\underline{h}=\underline{i}}\left(R_{\mathbb{Y}}\right)_{\underline{j}} \otimes\left(R_{\mathbb{Y}}\right)_{\underline{h}}\right)
$$

we have the $\mathbb{Z}^{k}$-homogeneous ideal $J=\operatorname{ker}(\mu)$, where $\mu: R_{\mathbb{Y}} \otimes_{K} R_{\mathbb{Y}} \rightarrow R_{\mathbb{Y}}$ is the multihomogeneous $R_{\mathbb{Y}}$-linear map given by $\mu(f \otimes g)=f g$. A $\mathbb{Z}^{k}$-homogeneous system of generators of $J$ is

$$
\left\{x_{i j_{i}} \otimes 1-1 \otimes x_{i j_{i}} \mid 1 \leq i \leq k, 0 \leq j_{i} \leq n_{i}\right\} .
$$


Definition 3.1. The $\mathbb{Z}^{k}$-graded $R_{Y}$-module $\Omega_{R_{Y} / K}^{1}=J / J^{2}$ is called the module of Kähler differentials of $R_{\mathbb{Y}} / K$. The $\mathbb{Z}^{k}$-homogeneous $K$-linear map $d_{R_{Y} / K}: R_{\mathbb{Y}} \rightarrow \Omega_{R_{\mathbb{Y}} / K}^{1}$ given by $f \mapsto f \otimes 1-1 \otimes f+J^{2}$ satisfies universal property. We call $d$ the universal derivation of $R_{\mathbb{Y}} / K$.

More generally, for any $\mathbb{Z}^{k}$-graded $K$-algebra $T / S$ we can define in the same way the Kähler differential module $\Omega_{T / S}^{1}$, and the universal derivation of $T / S$ (cf. [6, Section 2]).

Remark 3.2. (a) The $\mathbb{Z}^{k}$-graded $S$-module $\Omega_{S / K}^{1}$ has the representation

$$
\Omega_{S / K}^{1}=\bigoplus_{i=1}^{k} \bigoplus_{j=0}^{n_{i}} S d X_{i j} \cong S^{n_{1}+1}\left(-e_{1}\right) \oplus \cdots \oplus S^{n_{k}+1}\left(-e_{k}\right) .
$$

(b) If $I_{1}, I_{2}$ are $\mathbb{Z}^{k}$-homogeneous ideals of $S$, then

$$
I_{1} \Omega_{S / K}^{1} \cap I_{2} \Omega_{S / K}^{1}=\left(I_{1} \cap I_{2}\right) \Omega_{S / K}^{1}
$$

(see [7, Chapter 3, §7, Theorem 7.4(i)]).

Some following properties of the module of Kähler differentials follows from [6, Propositions 4.12 and 4.13].

Proposition 3.3. Let $d I_{Y}$ be the submodule of $\Omega_{S / K}^{1}$ generated by $\left\{d F \mid F \in I_{Y}\right\}$.

(a) There is an isomorphism of $\mathbb{Z}^{k}$-graded $R_{\mathbb{Y}}$-modules

$$
\Omega_{R_{Y} / K}^{1} \cong \Omega_{S / K}^{1} /\left(d I_{Y}+I_{Y} \Omega_{S / K}^{1}\right) .
$$

(b) The set $\left\{d x_{i j_{i}} \mid 1 \leq i \leq k, 0 \leq j_{i} \leq n_{i}\right\}$ is a $\mathbb{Z}^{k}$-homogeneous system of generators of $\Omega_{R_{Y} / K}^{1}$.

Example 3.4. Let $\mathbb{X}=\{P\}$ be the set of only one point in $\mathbb{P}^{n_{1}} \times \cdots \times \mathbb{P}^{n_{k}}$ where $P=[1$ : $0: \cdots: 0] \times \cdots \times[1: 0: \cdots: 0]$. We have $I_{P}=\left\langle X_{i j_{i}} \mid 1 \leq i \leq k, 1 \leq j \leq n_{i}\right\rangle$ and $R_{\mathbb{X}}=K\left[x_{10}, \ldots, x_{k 0}\right]$. Also, we see that

$$
I_{P} / I_{P}^{2} \cong R_{\mathbb{X}}^{n_{1}}\left(-e_{1}\right) \oplus \cdots \oplus R_{\mathbb{X}}^{n_{k}}\left(-e_{k}\right)
$$

and

$$
\Omega_{R_{\mathbb{X}} / K}^{1}=\left\langle d x_{i 0} \mid 1 \leq i \leq k\right\rangle \cong R_{\mathbb{X}}\left(-e_{1}\right) \oplus \cdots \oplus R_{\mathbb{X}}\left(-e_{k}\right) .
$$

Moreover, we get the exact sequence of $\mathbb{Z}^{k}$-graded $R_{\mathbb{X}}$-modules

$$
0 \longrightarrow I_{P} / I_{P}^{2} \longrightarrow R_{\mathbb{X}}^{n_{1}+1}\left(-e_{1}\right) \oplus \cdots \oplus R_{\mathbb{X}}^{n_{k}+1}\left(-e_{k}\right) \longrightarrow \Omega_{R_{\mathbb{X}} / K}^{1} \longrightarrow 0 .
$$

In this case the Hilbert function of $\Omega_{R_{\mathbb{X}} / K}^{1}$ satisfies

$$
\mathrm{HF}_{\Omega_{R_{\mathbb{X}}^{1} / K}^{1}}(\underline{i})=\mathrm{HF}_{\mathbb{X}}\left(\underline{i}-e_{1}\right)+\cdots+\mathrm{HF}_{\mathbb{X}}\left(\underline{i}-e_{k}\right) .
$$

In general, we have a presentation of module of Kähler differential as follows.

Theorem 3.5. Let $\mathbb{Y}=m_{1} P_{1}+\cdots+m_{s} P_{s}$ be a fat point scheme in $\mathbb{P}^{n_{1}} \times \cdots \times \mathbb{P}^{n_{k}}$, and let $\mathbb{V}$ be the thickening of $\Psi$. Then we have the exact sequence of $\mathbb{Z}^{k}$-graded $R_{\mathbb{Y}}$-modules

$$
0 \longrightarrow I_{\mathbb{Y}} / I_{\mathbb{V}} \longrightarrow R_{\mathbb{Y}}^{n_{1}+1}\left(-e_{1}\right) \oplus \cdots \oplus R_{\mathbb{Y}}^{n_{k}+1}\left(-e_{k}\right) \longrightarrow \Omega_{R_{\mathbb{Y}} / K}^{1} \longrightarrow 0 .
$$


To prove Theorem 3.5, we will first require the following lemma.

Lemma 3.6. Let $P=[1: 0: \cdots: 0] \times \cdots \times[1: 0: \cdots: 0]$ be a point of $\mathbb{P}^{n_{1}} \times \cdots \times \mathbb{P}^{n_{k}}$, let $m \geq 1$, and let $F \in I_{P}^{m} \backslash I_{P}^{m+1}$ be a $\mathbb{Z}^{k}$-homogeneous polynomial of degree $\underline{i} \in \mathbb{Z}^{k}$. Then we have $d F \notin I_{P}^{m} \Omega_{S / K}^{1}$.

Proof. Observe that $I_{P}^{m+1}=\left\langle X_{i j_{i}} \mid 1 \leq i \leq k, 1 \leq j \leq n_{i}\right\rangle^{m+1}$. Let $N=n_{1}+\cdots+n_{k}$. For $\underline{j} \in \mathbb{N}^{k}$ and $t \geq 1$, we set

$$
\Gamma_{\underline{j}, t}=\left\{\begin{array}{ll}
\alpha=\left(\alpha_{1}, \ldots, \alpha_{k}\right) \in \mathbb{N}^{N} \mid \begin{array}{l}
|\alpha|=t,\left(\left|\alpha_{1}\right|, \ldots,\left|\alpha_{k}\right|\right) \leq j \\
\alpha_{l}=\left(\alpha_{l 1}, \ldots, \alpha_{l n_{l}}\right), 1 \leq l \leq k
\end{array}
\end{array}\right\}
$$

Since $F \in I_{P}^{m}$, the polynomial $F$ can be written as

$$
F=\sum_{\alpha \in \Gamma_{i, m+1}} X_{11}^{\alpha_{11}} \cdots X_{1 n_{1}}^{\alpha_{1 n_{1}}} \cdots X_{k 1}^{\alpha_{k 1}} \cdots X_{k n_{k}}^{\alpha_{k n_{k}}} F_{\alpha}+\sum_{\beta \in \Gamma_{i, m}} X_{11}^{\beta_{11}} \cdots X_{1 n_{1}}^{\beta_{1 n_{1}}} \cdots X_{k 1}^{\beta_{k 1}} \cdots X_{k n_{k}}^{\beta_{k n_{k}}} G_{\beta}
$$

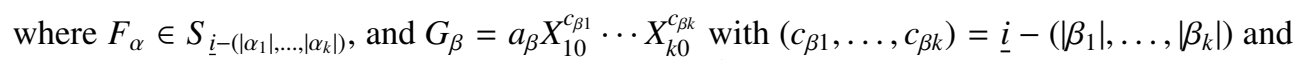
$a_{\beta} \in K$. It follows from $F \notin I_{P}^{m+1}$ that there exists $\widetilde{\beta} \in \Gamma_{\underline{i}, m}$ such that $a_{\widetilde{\beta}} \neq 0$.

In order to prove $d F \notin I_{P}^{m} \Omega_{S / K}^{1}$, it suffices to show that

$$
\omega=\sum_{\beta \in \Gamma_{\underline{\underline{i}, m}}} G_{\beta} d\left(X_{11}^{\beta_{11}} \cdots X_{1 n_{1}}^{\beta_{1 n_{1}}} \cdots X_{k 1}^{\beta_{k 1}} \cdots X_{k n_{k}}^{\beta_{k n_{k}}}\right) \notin I_{P}^{m} \Omega_{S / K}^{1} .
$$

Write $G_{\widetilde{\beta}}=a_{\widetilde{\beta}} \cdot T$ with the term $T=X_{10}^{c_{\widetilde{\beta} 1}} \cdots X_{k 0}^{c_{\overline{\beta k}}}$, and set

$$
\widetilde{\Gamma}=\left\{\beta \in \Gamma_{\underline{i}, m} \mid a_{\beta} \neq 0, G_{\beta}=a_{\beta} T\right\} .
$$

Clearly, $\widetilde{\beta} \in \widetilde{\Gamma} \neq \emptyset$. Let $(i, j)$ be the smallest tuple w.r.t. Lex such that there exists $\beta \in \widetilde{\Gamma}$ such that $b_{i j} \neq 0$. Write $\widetilde{\Gamma}=\left\{\widetilde{\beta}^{1}, \ldots, \widetilde{\beta}^{s}\right\}$ such that $\widetilde{\beta}^{1} \leq_{\text {Lex }} \cdots \leq_{\text {Lex }} \widetilde{\beta}^{s}$. Then $\widetilde{\beta}_{i j}^{1} \leq \cdots \leq \widetilde{\beta}_{i j}^{s}$. Set $\varrho=\min \left\{\widetilde{\beta}_{i j}^{l} \mid \widetilde{\beta}_{i j}^{l}>0,1 \leq l \leq s\right\}$. We have

$$
\begin{aligned}
\widetilde{\omega}:= & \sum_{\beta \in \widetilde{\Gamma}} a_{\beta} T d\left(X_{11}^{\beta_{11}} \cdots X_{1 n_{1}}^{\beta_{1 n_{1}}} \cdots X_{k 1}^{\beta_{k 1}} \cdots X_{k n_{k}}^{\beta_{k n_{k}}}\right) \\
= & \sum_{\beta \in \widetilde{\Gamma}} a_{\beta} T d\left(X_{i j}^{\beta_{i j}} \cdots X_{i n_{i}}^{\beta_{i n_{i}}} \cdots X_{k 1}^{\beta_{k 1}} \cdots X_{k n_{k}}^{\beta_{k n_{k}}}\right) \\
= & \sum_{\beta \in \widetilde{\Gamma}, \beta_{i j} \neq \varrho} a_{\beta} T d\left(X_{i j}^{\beta_{i j}} \cdots X_{i n_{i}}^{\beta_{i n_{i}}} \cdots X_{k 1}^{\beta_{k 1}} \cdots X_{k n_{k}}^{\beta_{k n_{k}}}\right)+\sum_{\beta \in \widetilde{\Gamma}, \beta_{i j}=\varrho} a_{\beta} T d\left(X_{i j}^{\varrho} \cdots X_{i n_{i}}^{\beta_{i n_{i}}} \cdots X_{k 1}^{\beta_{k 1}} \cdots X_{k n_{k}}^{\beta_{k n_{k}}}\right) \\
& \stackrel{(\star)}{=} \sum_{\beta \in \widetilde{\Gamma}, \beta_{i j} \neq \varrho} a_{\beta} T d\left(X_{i j}^{\beta_{i j}} \cdots X_{i n_{i}}^{\beta_{i n_{i}}} \cdots X_{k 1}^{\beta_{k 1}} \cdots X_{k n_{k}}^{\beta_{k n_{k}}}\right) \\
& +\sum_{\beta \in \widetilde{\Gamma}, \beta_{i j}=\varrho} a_{\beta} T \varrho X_{i j}^{\varrho-1} X_{i j+1}^{\beta_{i j+1}} \cdots X_{i n_{i}}^{\beta_{i n_{i}}} \cdots X_{k 1}^{\beta_{k 1}} \cdots X_{k n_{k}}^{\beta_{k n_{k}}} d X_{i j} \\
& +\sum_{\beta \in \widetilde{\Gamma}, \beta_{i j}=\varrho} a_{\beta} T X_{i j}^{\varrho} d\left(X_{i j+1}^{\beta_{i j+1}} \cdots X_{i n_{i}}^{\beta_{i n_{i}}} \cdots X_{k 1}^{\beta_{k 1}} \cdots X_{k n_{k}}^{\beta_{k n_{k}}}\right) .
\end{aligned}
$$

Note that if $\beta, \beta^{\prime} \in \widetilde{\Gamma}, \beta \neq \beta^{\prime}$ and $\beta_{i j}=\beta_{i j}^{\prime}=\varrho$, then $\left(\beta_{i j+1}, \ldots, \beta_{k n_{k}}\right) \neq\left(\beta_{i j+1}^{\prime}, \ldots, \beta_{k n_{k}}^{\prime}\right)$. By Macaulay's Basis Theorem (cf. [5, Theorem 1.5.7]), we have

$$
\sum_{\beta \in \widetilde{\Gamma}, \beta_{i j}=\varrho} a_{\beta} T \varrho X_{i j}^{\varrho-1} X_{i j+1}^{\beta_{i j+1}} \cdots X_{i n_{i}}^{\beta_{i n_{i}}} \cdots X_{k 1}^{\beta_{k 1}} \cdots X_{k n_{k}}^{\beta_{k n_{k}}} d X_{i j} \notin I_{P}^{m} \Omega_{S / K}^{1}
$$


It follows that $\widetilde{\omega} \notin I_{P}^{m} \Omega_{S / K}^{1}$ (since in the right-hand side of $(\star)$ the first summand has $\beta_{i j} \neq \varrho$ and the last summand does not contain $d X_{i j}$ ). Therefore we get $\omega \notin I_{P}^{m} \Omega_{S / K}^{1}$, as we wanted to show.

We are now ready to state and prove the main result of this section.

Proof. Let $\varphi: I_{\mathbb{Y}} / I_{\mathbb{V}} \rightarrow \Omega_{S / K}^{1} / I_{Y} \Omega_{S / K}^{1}$ be the map given by $\varphi\left(F+I_{\mathbb{V}}\right)=d F+I_{Y} \Omega_{S / K}^{1}$ for all $F \in I_{Y}$. It is easy to check that the map $\varphi$ is well-defined, $\mathbb{Z}^{k}$-homogeneous of degree $(0, \ldots, 0)$, and $R_{\mathbb{Y}}$-linear. For any $\mathbb{Z}^{k}$-homogeneous element $F \in I_{\mathbb{Y}} \backslash I_{\mathbb{V}}$, we have $F \in$ $I_{P_{j}}^{m_{j}} \backslash I_{P_{j}}^{m_{j}+1}$ for some $j \in\{1, \ldots, s\}$. W.l.o.g. we may assume that $P_{j}=\left[\begin{array}{lll}1: 0 & : \cdots & \cdots\end{array}\right.$ $0] \times \cdots \times[1: 0: \cdots: 0]$. So, Lemma 3.6 yields $d F \notin I_{P_{j}}^{m_{j}} \Omega_{S / K}^{1}$. Since $I_{Y} \Omega_{S / K}^{1}=\bigcap_{j=1}^{s} I_{P_{j}}^{m_{j}} \Omega_{S / K}^{1}$ by Remark 3.2(b), we obtain $d F \notin I_{Y} \Omega_{S / K}^{1}$. Consequently, the map $\varphi$ is injective. Moreover, by Remark 3.2(a) and Proposition 3.3, we see that

$$
\Omega_{S / K}^{1} / I_{\mathbb{Y}} \Omega_{S / K}^{1} \cong R_{\mathbb{Y}}^{n_{1}+1}\left(-e_{1}\right) \oplus \cdots \oplus R_{\mathbb{Y}}^{n_{k}+1}\left(-e_{k}\right)
$$

and

$$
\left(\Omega_{S / K}^{1} / I_{Y} \Omega_{S / K}^{1}\right) / \operatorname{im}(\varphi) \cong \Omega_{S / K}^{1} /\left(d I_{Y}+I_{Y} \Omega_{S / K}^{1}\right) \cong \Omega_{R_{Y} / K}^{1} .
$$

Therefore the conclusion follows.

The following relation between the Hilbert function of $\Omega_{R_{Y} / K}^{1}$ and of $\mathbb{Y}$ and $\mathbb{V}$ is an immediate consequence of Theorem 3.5

Corollary 3.7. $\mathbb{Y}=m_{1} P_{1}+\cdots+m_{s} P_{s}$ be a fat point scheme in $\mathbb{P}^{n_{1}} \times \cdots \times \mathbb{P}^{n_{k}}$, and let $\mathbb{V}$ be the thickening of $\mathbb{Y}$. Then the Hilbert function of $\Omega_{R_{\mathbb{Y}} / K}^{1}$ satisfies

$$
\mathrm{HF}_{\Omega_{R_{Y} / K}^{1}}(\underline{i})=\left(n_{1}+1\right) \mathrm{HF}_{\mathbb{Y}}\left(\underline{i}-e_{1}\right)+\cdots+\left(n_{k}+1\right) \mathrm{HF}_{\mathbb{Y}}\left(\underline{i}-e_{k}\right)+\mathrm{HF}_{\mathbb{Y}}(\underline{i})-\mathrm{HF}_{\mathbb{V}}(\underline{i})
$$

for all $\underline{i} \in \mathbb{Z}^{k}$.

\section{References}

[1] G. de Dominicis and M. Kreuzer, Kähler differentials for points in $\mathbb{P}^{n}$, J. Pure Appl. Alg. 141, 153-173 (1999).

[2] E. Guardo, M. Kreuzer, T. N. K. Linh, and L.N. Long, Kähler differentials for fat point schemes in $\mathbb{P}^{1} \times \mathbb{P}^{1}$, Submitted (2018).

[3] E. Guardo and A. Van Tuyl, Fat Points in $\mathbb{P}^{1} \times \mathbb{P}^{1}$ and their Hilbert functions, Canad. J. Math. 56 , no. 4, 716-741 (2004).

[4] M. Kreuzer, T. N. K. Linh, and L. N. Long, Kähler differentials and Kähler differents for fat point schemes, J. Pure Appl. Algebra 219, 4479-4509 (2015).

[5] M. Kreuzer and L. Robbiano, Computational Commutative Algebra 1, (Springer Verlag, Heildelberg, 2000).

[6] E. Kunz, Kähler Differentials (Adv. Lectures Math., Wieweg Verlag, Braunschweig, 1986).

[7] H. Matsumura, Commutative Ring Theory (Cambridge University Press, Cambrige, 1986).

[8] J. Sidman and A. Van Tuyl, Multigraded regularity: Syzygies and fat points, Beiträge zur Algebra und Geometrie 47, 1-22 (2006). 